\title{
PUT at SemEval-2016 Task 4: The ABC of Twitter Sentiment Analysis
}

\author{
Mateusz Lango and Dariusz Brzezinski and Jerzy Stefanowski \\ Institute of Computing Science, Poznan University of Technology \\ 60-965 Poznań, Poland \\ \{mlango, dbrzezinski,jstefanowski\}@cs.put.edu.pl
}

\begin{abstract}
This paper describes a classification system that participated in SemEval-2016 Task 4: Sentiment Analysis in Twitter. The proposed approach competed in subtasks A, B, and C, which involved tweet polarity classification, tweet classification according to a two-point scale, and tweet classification according to a five-point scale. Our system is based on an ensemble consisting of Random Forests, SVMs, and Gradient Boosting Trees, and involves the use of a wide range of features including: $n-$ grams, Brown clustering, sentiment lexicons, Wordnet, and part-of-speech tagging. The proposed system achieved $14^{\text {th }}, 6^{\text {th }}$, and $3^{\text {rd }}$ place in subtasks $\mathrm{A}, \mathrm{B}$, and $\mathrm{C}$, respectively.
\end{abstract}

\section{Introduction}

In recent years, sentiment analysis (Liu, 2012) has become a common yardstick for many new text mining algorithms. This trend is a direct result of the rapid growth of social media, where users express their views and opinions regarding a wide range of topics. As a result, social networks like Twitter have become a crucial resource in product design, assessing marketing campaigns, and detecting news bursts (Liu, 2012; Mathioudakis and Koudas, 2010).

However, while the merits of resources such as Twitter are evident, there are several difficulties with the use of social media data. In contrast to classical sentiment analysis methods, which were originally designed for dealing with well-written product reviews, texts from social media often contain misspellings, letter substitutions, ambiguities, nonstandard abbreviations, and improper use of gram- mar (Sarker et al., 2015). Furthermore, resources such as Twitter generate thousands of new texts per second and introduce challenges characteristic for stream processing (Krempl et al., 2014). Moreover, the limited length of these texts makes classical ngram feature vectors extremely sparse, which in turn hinders generalization abilities of classification algorithms. Finally, sentiments are usually unevenly distributed (Kiritchenko et al., 2014), resulting in class imbalance and, therefore, additional difficulties for classifiers (He and Garcia, 2009).

To promote research in this area, Task 4 of SemEval-2016 was devoted to sentiment analysis in Twitter. The task consisted of five subtasks involving standard classification, ordinal classification, and distribution estimation; for a more detailed description see (Nakov et al., 2016).

In this paper, we present our approach to learn a classification system which participated in subtasks A, B, and C of SemEval-2016 Sentiment Analysis in Twitter. The proposed approach combines Random Forests, Support Vector Machines, and Gradient Boosting Trees, trained on a wide range of lexical and semantic features including: n-grams, kgrams, Brown clustering, sentiment lexicons, SentiWordNet, and part of speech tagged 1-grams. These components were carefully combined and optimized to create a separate version of the system for each of the tackled subtasks.

In the following sections, we describe each group of features used in our system. Moreover, we explain the details of the proposed classification algorithm with respect to each realized subtask. Finally, we conclude the paper with a discussion on the ob- 
tained results, importance of each feature group, and possible lines of future research.

\section{Basic Features}

We briefly describe the features used in our system. The same set of developed features was used in all three subtasks our algorithms participated in. However, for some of the component classifiers we additionally performed feature selection using a filter method based on the F-statistic. Details on this subject will be discussed later.

\subsection{Preprocessing}

Prior to extracting features, we performed standard natural language processing procedures to clean the data. First, each tweet was tokenized into words, hashtags, punctuation marks, and special symbols. Next, tokens were lemmatized by NLTK WordNetLemmatizer ${ }^{1}$ to unify different versions of the same words. Subsequently, certain words were removed based on a hand-crafted stop list. Finally, certain symbols (urls, hashtags, numbers, percentages, prices, dates, hours) that occurred less than five times in the dataset were grouped according to their meaning, and those tokens that could not be grouped were removed from the training data.

\subsection{Word n-grams}

The first feature set consisted of word n-grams, i.e., sequences of $n$ continuous words in a text segment. For our system, we generated 1-, 2-, 3-, 4-, and 5grams based on all available tweet messages.

\subsection{Negation n-grams}

In addition to traditional n-grams, we also utilized n-grams in negation context (Remus, 2013). Negation n-grams are sequences of words that appear in a negated context. Negations were discovered based on "not" and "n't" tokens, and a negated context was defined as a set of words falling between a negation and a "terminal" punctuation symbol $\{., ;,,,, !, ?\}$. We used 1- and 2-negation-grams in our system.

\subsection{Character k-grams}

Another group of features was created by generating character k-grams. Character k-grams were cre-

\footnotetext{
${ }^{1}$ http://www.nltk.org/api/nltk.stem.html
}

ated by extracting sequences of $k$ continuous characters from each word. To distinguish charactergrams from word-grams, we will refer to character sequences as k-grams. We used 3-, 4-, and 5character-grams as features.

\subsection{POS 1-grams}

Another set of $n$-grams was created by using a partof-speech tagger. This approach combines words with the part of speech they represent, in an attempt to distinguish different meanings of the same word. In our system, we used the NLTK PerceprtronTagger $^{2}$ to add concatenated \{word, part-of-speech pairs as features.

\subsection{Sentiment Lexicons}

A major group of features used in our system was formed by sentiment scores, which were created by summing word-sentiment associations for a given tweet. More precisely, for each tweet we counted the number of words conveying each sentiment defined in a given lexicon. We used this procedure for four sentiment lexicons: the NRC emotion lexicon (Mohammad and Turney, 2013), Hu and Liu Opinion lexicon (Hu and Liu, 2004), the Multi-perspective Question Answering corpus (Wiebe et al., 2005), and SentiWordNet (Baccianella et al., 2010).

The NRC emotion lexicon is a list of words and their associations with eight emotions (anger, fear, anticipation, trust, surprise, sadness, joy, and disgust) and two sentiments (negative and positive). Combined this gives a total of ten real valued sentiment scores, which were added to our feature set.

The Opinion Lexicon, assembled by $\mathrm{Hu}$ and Liu, consists of two lists: one containing positive and one containing negative words. Because intensities of these two sentiments are not specified, we counted the occurrences of lexicon words in each tweet to create two sentiment scores.

The Multi-perspective Question Answering (MPQA) corpus contains four sentiment word lists: positive, negative, both, and neutral. As with the Opinion Lexicon, we counted the occurrences of each in-lexicon word to create four additional features.

Finally, the SentiWordNet is a sentiment tagged

\footnotetext{
${ }^{2}$ http://www.nltk.org/api/nltk.tag.html
} 
wordnet. We used this network to find synsets (semantical equivalents) of words and used their sentiment scores as features.

\subsection{Hashtag Lexicon}

An interesting addition to the aforementioned word lexicons was the use of the NRC Hashtag Affirmative/Negated Context Sentiment Lexicon (Kiritchenko et al., 2014). This lexicon contains a realvalued sentiment score associated with single words and 2-grams designed specifically for Twitter. For each tweet we calculated the minimal, maximal, and mean sentiment score based on all words in a tweet.

\subsection{Brown Clustering}

Our final set of features was created using Brown clustering (Brown et al., 1992). Brown clustering is a form of hierarchical clustering of words based on the contexts in which they occur. We used a precompiled clustering of English tweets into 1000 clusters provided by Owoputi et al. (2013).

\section{Classification}

\subsection{Multi-class classification (subtask A)}

The goal of subtask A was to correctly classify tweets into three classes: positive, neutral, and negative. Macro-averaged $F_{1}$ score over the positive and negative class was used as an evaluation metric. The provided training set consisted of 5459 tweets $^{3}$ and the test set, which was used for internal model verification and validation, consisted of 1806 tweets.

Gradient Boosting Trees (Friedman, 2001) is a popular classifier which combines the idea of a boosting ensemble and gradient descent optimization. We have chosen it, because it proved to work well in many data mining competitions and on a variety of problems. GBT are also robust to very sparse features, which makes them a good choice for tweet classification.

In our system we used GBT with softmax as the loss function, the maximum depth of a single tree was set to 40 and no tree pruning was performed afterwards. To prevent overfitting the L2 regularization factor was added to the optimization func-

\footnotetext{
${ }^{3}$ The dataset provided by task organizers was a little bit bigger, but we report the number of tweets which we were able to download successfully.
}

tion. Additionally, to increase the diversity of the model, each ensemble component was trained on a subset of features. Each subset was constructed using randomly chosen $40 \%$ of the features. Furthermore, each tree component was trained on a random sample of the training set which contained $80 \%$ of the examples.

From the training set $10 \%$ of examples were extracted to form a validation set. This additional dataset was used for verification of the early stopping condition. After learning every new tree, the performance of the whole classifier was verified on the validation set in terms of macro-averaged $F_{1}$ score. The lack of improvement during 30 iterations triggered the early stopping condition and terminated the ensemble construction. GBT was always fitted until the early stopping condition was met, without any constraint on the maximal number of ensemble components ${ }^{4}$.

During initial experiments we discovered, that the classifier made wrong predictions on negative and neutral examples more often than on instances belonging to the positive class. The trained model suffered from class imbalance, which often leads to generalization problems of many classification techniques (He and Garcia, 2009). Indeed, the dataset in this subtask contains 2804 positive examples (51\%) together with only 781 negative (14\%) and 1874 neutral $(34 \%)$ examples.

To overcome this problem, inspired by solutions in the field of cost-sensitive learning ( $\mathrm{He}$ and Garcia, 2009), we assigned each instance a weight $w=$ $1 /\left(c \cdot\left|C_{i}\right|\right)$ where $\left|C_{i}\right|$ is the number of examples belonging to the true class of the $i$-th example in the training set and $c$ is the total number of classes (in this subtask $c=3$ ). The use of such instance weights in the loss function ensures that each class is equally important for optimization, because the sums of example weights for each class are equal.

We also tested Random Forests (Breiman, 2001) and linear Support Vector Machines (Cortes and Vapnik, 1995) classifiers. As preliminary experiments showed that the performance of Random Forests and SVM was sensitive to the increasing number of features, we decided to carry out addi-

\footnotetext{
${ }^{4}$ In practice we always set the maximum number of iterations to a big number (10000).
} 
tional feature selection. Hence, we trained them on 5000 best features selected by the $\mathrm{F}$ value of ANOVA which improved micro-averaged $F_{1}$ and also had a positive influence on training time. The best results for Random Forest, according to macroaveraged $F_{1}$, were achieved when each leaf of a single tree was enforced to contain at least three examples, the number of trees was equal to 5000 and instance weighting (as described above) was used. Also SVM gave best results with instance weighting. Despite the fact that both Random Forests and SVMs achieved results that were a little worse than GBT (macro-averaged $F_{1}$ score was about $3 \%$ lower for both of them) we decided to use them to refine predictions of GBT.

Finally, our classification system is a heterogeneous multiple-classifier consisting of three different components: Random Forests, Gradient Boosting Trees, and Support Vector Machines. Each of them is trained on the same training set and the final classification of the ensemble is a result of simple majority voting. We use a well-known scikitlearn (Pedregosa et al., 2011) implementation of Random Forests, and SVMs in Python as well as a very effective Gradient Boosting Trees implementation from the XGBoost library ${ }^{5}$.

\subsection{Binary classification (subtask B)}

The goal of subtask B also involved the classification of tweets, however, only two classes (positive and negative) were considered. Just as in subtask A, the dataset was highly imbalanced: only $17 \%$ (679) of examples were negative.

In subtask A we could not use more advanced methods for tackling class imbalance since most of them are designed for binary classification only. One of such techniques is Roughly Balanced Bagging (Hido et al., 2009), which proved to give the best results among extensions of bagging for class imbalance (Błaszczyński and Stefanowski, 2015).

RBBag learns each base classifier on a random sample of the training set and then the final class prediction is a result of averaging predictions of components. The main difference between classical bagging and RBBag is its specialized sampling scheme. First, the training set is divided into two

\footnotetext{
${ }^{5}$ https://github.com/dmlc/xgboost
}

subsets, each containing examples from only one class. From the subset containing minority examples, RBBag creates a classical bootstrap sample which contains $N$ instances, where $N$ is the number of minority examples in the training set. To this sample, $M$ majority examples are added randomly where $M$ is not the number of majority examples, but it is taken from a negative binomial distribution with parameters $p=0.5$ and $n=N$.

We used Roughly Balanced Bagging with GBT as the base classifier. All parameters of GBT were set just like described in section 3.1, however during experiments different learning rates and regularization factors were selected. Additionally, RBBag was tested with 5, 7, 15 and 30 base classifiers, but the best results were obtained for 7 GBTs, which confirms earlier observations of Lango and Stefanowski (2015) that RBBag does not require many components to achieve good performance.

\subsection{Ordinal classification (subtask C)}

Subtask C concentrated on classifying tweets into 5 classes: very negative, negative, neutral, positive and very positive. Since the order in the classes is established, this subtask can be considered as an ordinal classification problem.

We implemented an ensemble algorithm described by Frank and Hall (2001), which decomposes ordinal classification into several binary classification problems. Each classifier is trained on the same training set, but the class label of every example is changed by the function $I\left(x_{\text {class }}>i\right)$ where $I()$ is an indicator function, $x_{\text {class }}$ is a class of a given example and $i$ is the reference class. The reference class for the first classifier is "very negative", for the second "negative" etc. Finally, we have four classifiers and each of them returns the likelihood of a positive response to the question "is the class of the analyzed example higher than the reference class". The final set of likelihoods can be easily transformed to the likelihood of every class.

Again, we used GBT as a base classifier with the same setup as described in section 3.1. However, we also tested linear SVM, Random Forests, Factorization Machines (Rendle, 2010) and transductive SVMs (Joachims, 1999). Linear SVM achieved results very similar to GBT in terms of macroaveraged mean absolute error $\left(M A E^{M}\right)$. Despite 
this fact, during the analysis of responses of both classifiers on the test set, for several examples we discovered significant differences in responses (e.g. "very negative" vs "positive"). Since both models performed almost equally good and $M A E^{M}$ highly punishes significant differences between classes on the ordinal scale, we decided to create a metaclassifier from these two models. In our ensemble the final prediction is an average of predictions of GBT and SVM-based models, which is rounded towards the decision of the GBT-based model (since its $M A E^{M}$ score was a little higher).

\section{Results and feature analysis}

This section includes the experimental results of our system for all three sub-tasks. We present the scores and ranks achieved by our system followed by a discussion on the relative importance of the proposed features.

The evaluation metric was different in each subtask (Nakov et al., 2016). For subtask A, it was required to optimize the macro-averaged $F_{1}$-score $\left(F_{1}^{M}\right)$ calculated over the positive and negative classes. In subtask B, the goal was to achieve a high macro-averaged recall $\left(R^{M}\right)$, while subtask $\mathrm{C}$ took into account a macro-averaged mean absolute error $\left(M A E^{M}\right)$. Table 4 presents the overall performance of our system.

\begin{tabular}{|l|l|r|r|r|}
\hline Subtask & Metric & Our score & Best score & Rank \\
\hline A & $F_{1}^{M}$ & 0.576 & 0.633 & 14 \\
B & $R^{M}$ & 0.763 & 0.797 & 6 \\
C & $M A E^{M}$ & 0.860 & 0.719 & 3 \\
\hline
\end{tabular}

Table 1: Overall performance of the system.

We also performed an analysis of feature importance using one trained Gradient Boosting Trees classifier (GBT). For this classifier the feature importance can be easily measured by observing the increase of purity while performing splits on a particular feature, following an approach from (Breiman and Friedman, 1984).

In subtasks $\mathrm{A}$ and $\mathrm{C}$ we used a meta-classifier of many different algorithms, so the results would not accurately reflect the feature importance in the whole system. Hence, we decided to run this experiment on the dataset from subtask B only.

Table 2 presents 15 features with the highest rel- ative importance in our classifier. The most important feature was the mean of word sentiments in a tweet according to the NRC Hashtag Lexicon (the maximum word sentiment on this lexicon is also pretty high in the ranking). Other lexicon features, based on the Opinion Lexicon and SentiWordNet, also achieved high relative importance. Note that many features with high importance come from Brown clustering and k-grams.

In Table 3, we present results of feature importance aggregated in groups. The most important features are those created from character k-grams and their total relative importance is almost $70 \%$. The contribution of features created from Brown clustering, negated $\mathrm{n}$-grams and from $\mathrm{n}$-grams with part-ofspeech tags is also very significant. The importance of the rest of the features sums up to only $10 \%$. The poor results of lexicon-based features can be justified by the fact that the number of features in these groups is very small (from 2 to 8 features).

\begin{tabular}{|l|r|}
\hline Feature name & Rel. impor. [\%] \\
\hline NRC Hashtag Lexicon: mean & 0.79 \\
Brown cluster: 01110110 & 0.73 \\
SentiWordNet: sum of negative & 0.63 \\
5 k-gram: "d \&am" & 0.55 \\
Brown cluster: 1110011001111 & 0.49 \\
NRC Hashtag Lexicon: max & 0.48 \\
Opinion Lexicon: negative & 0.47 \\
Brown cluster: 111101011101 & 0.42 \\
3 k-gram "ok" & 0.41 \\
4 k-gram "nor" & 0.40 \\
Brown cluster: 0100100 & 0.38 \\
3 k-gram “NY" & 0.35 \\
2 n-gram: not against & 0.35 \\
Brown cluster: 111101111100100 & 0.34 \\
5 k-gram " Anth" & 0.34 \\
\hline
\end{tabular}

Table 2: Relative feature importances (\%) of top 15 features.

For Random Forests and SVM we used feature selection according to the F-statistics. We analyzed how features selected by this approach relate to importances estimated by GBT.

Surprisingly, feature importances estimated by the F-statistic and GBT are quite coherent. Almost $80 \%$ of features selected by the F-statistic were character-grams, $12 \%$ of features were negated ngrams, and features from POS constituted 3,9\% of all selected features. The main difference between these two methods is that the F-statistic selected 


\begin{tabular}{|l|r|}
\hline Feature group & Rel. impor. [\%] \\
\hline 5 character-gram & 26.03 \\
4 character-gram & 21.75 \\
3 character-gram & 21.74 \\
Brown clusters & 6.92 \\
Negated 1-gram & 6.62 \\
1-gram + POS & 4.24 \\
Negated + 2-gram & 3.48 \\
1-gram & 2.69 \\
2-gram & 1.87 \\
NRC Hashtag Lexicon & 1.49 \\
SentiWordNet & 1.00 \\
NRC Lexicon & 0.93 \\
Opinion Lexicon & 0.62 \\
3-gram & 0.34 \\
MPQA corpus & 0.25 \\
4-gram & 0.03 \\
\hline
\end{tabular}

Table 3: Relative feature importances (\%) for features groups.

only one feature from Brown clustering. However, once again simple n-grams were used very rarely ( $2 \%$ of all selected features). This result, together with earlier observations from importances estimated by GBT, seem to show that features created from character-grams are superior to those created by word-grams. It is also worth mentioning that the entire GBT model used only 3579 features, which is an indicator of its feature selection abilities.

\section{Conclusions and Future Work}

Our system achieved relatively good performance in SemEval-2016 Task 4: Sentiment Analysis in Twitter. Among 34 participants of subtask A we reached rank 14 , we took $6^{\text {th }}$ place among 19 competitors in subtask $B$, and won $3^{\text {rd }}$ place in subtask $C$ where 11 teams competed. The analysis of features used by our system shows that character-grams seem to perform better than word n-grams for Twitter's shorttext messages. Furthermore, results obtained by Gradient Boosting Trees in our system confirmed good feature filtering capabilities of this algorithm.

One possible way to further improve our system could be to transfer features selected by GBT to other classifiers (e.g. SVM). Another possible line of the future research is the development of new features based on character-grams, such as negated character-grams or character-gram lexicons.

\section{Acknowledgments}

This research was partially funded by the Polish National Science Center under Grant No. DEC2013/11/B/ST6/00963.

\section{References}

Stefano Baccianella, Andrea Esuli, and Fabrizio Sebastiani. 2010. Sentiwordnet 3.0: An enhanced lexical resource for sentiment analysis and opinion mining. In Proceedings of the International Conference on Language Resources and Evaluation.

Jerzy Błaszczyński and Jerzy Stefanowski. 2015. Neighbourhood sampling in bagging for imbalanced data. Neurocomputing, 150, Part B:529-542.

Leo Breiman and Jerome H. Friedman. 1984. Classification and regression trees. Chapman \& Hall, New York.

Leo Breiman. 2001. Random forests. Machine learning, 45(1):5-32.

Peter F. Brown, Vincent J. Della Pietra, Peter V. de Souza, Jennifer C. Lai, and Robert L. Mercer. 1992. Classbased n-gram models of natural language. Computational Linguistics, 18(4):467-479.

Corinna Cortes and Vladimir Vapnik. 1995. Support vector machine. Machine learning, 20(3):273-297.

Eibe Frank and Mark Hall. 2001. A simple approach to ordinal classification. In Proceedings of the 12th European Conference on Machine Learning, pages 145156.

Jerome H Friedman. 2001. Greedy function approximation: a gradient boosting machine. Annals of statistics, pages $1189-1232$.

Haibo He and Edwardo A Garcia. 2009. Learning from imbalanced data. Knowledge and Data Engineering, IEEE Transactions on, 21(9):1263-1284.

Shohei Hido, Hisashi Kashima, and Yutaka Takahashi. 2009. Roughly balanced bagging for imbalanced data. Statistical Analysis and Data Mining, 2(5-6):412-426.

Minqing $\mathrm{Hu}$ and Bing Liu. 2004. Mining and summarizing customer reviews. In Proceedings of the Tenth ACM SIGKDD International Conference on Knowledge Discovery and Data Mining, pages 168-177.

Thorsten Joachims. 1999. Transductive inference for text classification using support vector machines. In International Conference on Machine Learning (ICML), pages 200-209.

Svetlana Kiritchenko, Xiaodan Zhu, and Saif M. Mohammad. 2014. Sentiment analysis of short informal texts. Journal of Artificial Intelligence Research, 50:723-762. 
Georg Krempl, Indrè Žliobaitè, Dariusz Brzezinski, Eyke Hüllermeier, Mark Last, Vincent Lemaire, Tino Noack, Ammar Shaker, Sonja Sievi, Myra Spiliopoulou, and Jerzy Stefanowski. 2014. Open challenges for data stream mining research. SIGKDD Explorations, 16(1):1-10.

Mateusz Lango and Jerzy Stefanowski. 2015. Applicability of roughly balanced bagging for complex imbalanced data. In Proceedings of the 4th Workshop on New Frontiers in Mining Complex Patterns (NFMCP 2015), pages 62-73.

Bing Liu. 2012. Sentiment Analysis and Opinion Mining. Synthesis digital library of engineering and computer science. Morgan \& Claypool.

Michael Mathioudakis and Nick Koudas. 2010. Twittermonitor: Trend detection over the twitter stream. In Proceedings of the 2010 ACM SIGMOD International Conference on Management of Data, pages 11551158.

Saif Mohammad and Peter D. Turney. 2013. Crowdsourcing a word-emotion association lexicon. Сотриtational Intelligence, 29(3):436-465.

Preslav Nakov, Alan Ritter, Sara Rosenthal, Veselin Stoyanov, and Fabrizio Sebastiani. 2016. SemEval-2016 task 4: Sentiment analysis in Twitter. In Proceedings of the 10th International Workshop on Semantic Evaluation.

Olutobi Owoputi, Brendan O'Connor, Chris Dyer, Kevin Gimpel, Nathan Schneider, and Noah A. Smith. 2013. Improved part-of-speech tagging for online conversational text with word clusters. In Proceedings of Human Language Technologies: Conference of the North American Chapter of the Association of Computational Linguistics, pages 380-390.

F. Pedregosa, G. Varoquaux, A. Gramfort, V. Michel, B. Thirion, O. Grisel, M. Blondel, P. Prettenhofer, R. Weiss, V. Dubourg, J. Vanderplas, A. Passos, D. Cournapeau, M. Brucher, M. Perrot, and E. Duchesnay. 2011. Scikit-learn: Machine learning in Python. Journal of Machine Learning Research, 12:28252830.

Robert Remus. 2013. Modeling and representing negation in data-driven machine learning-based sentiment analysis. In Proceedings of the First International Workshop on Emotion and Sentiment in Social and Expressive Media: approaches and perspectives from AI (ESSEM 2013), pages 22-33.

Steffen Rendle. 2010. Factorization machines. In Proceedings of the 10th International Conference on Data Mining (ICDM), pages 995-1000.

Abeed Sarker, Azadeh Nikfarjam, Davy Weissenbacher, and Graciela Gonzalez. 2015. Diegolab: An approach for message-level sentiment classification in twitter. In
Proceedings of the 9th International Workshop on Semantic Evaluation, pages 510-514.

Janyce Wiebe, Theresa Wilson, and Claire Cardie. 2005. Annotating expressions of opinions and emotions in language. Language Resources and Evaluation, 39(23):165-210. 